\title{
MOST photometry of the roAp star 10 Aquilae ${ }^{\star}$
}

\author{
D. Huber ${ }^{1}$, H. Saio ${ }^{2}$, M. Gruberbauer ${ }^{1}$, W. W. Weiss ${ }^{1}$, J. F. Rowe ${ }^{3}$, M. Hareter ${ }^{1}$, T. Kallinger ${ }^{1}$, P. Reegen ${ }^{1}$, \\ J. M. Matthews ${ }^{3}$, R. Kuschnig ${ }^{1}$, D. B. Guenther ${ }^{4}$, A. F. J. Moffat ${ }^{5}$, S. Rucinski ${ }^{6}$, D. Sasselov ${ }^{7}$, and G. A. H. Walker ${ }^{3}$ \\ 1 Institute for Astronomy (IfA), University of Vienna, Türkenschanzstrasse 17, 1180 Vienna, Austria \\ e-mail: huber@astro.univie.ac.at \\ 2 Astronomical Institute, Graduate School of Science, Tohoku University, Sendai 980-8578, Japan \\ 3 Dept. of Physics and Astronomy, University of British Columbia, 6224 Agricultural Road, Vancouver, BC V6T 1Z1, Canada \\ 4 Dept. of Astronomy and Physics, St. Mary's University, Halifax, NS B3H 3C3, Canada \\ 5 Dépt. de physique, Univ. de Montréal, CP 6128, Succ. Centre-Ville, Montréal, QC H3C 3J7, Canada \\ 6 Dept. of Astronomy \& Astrophysics, David Dunlop Obs., Univ. Toronto, PO Box 360, Richmond Hill, ON L4C 4Y6, Canada \\ 7 Harvard-Smithsonian Center for Astrophysics, 60 Garden Street, Cambridge, MA 02138, USA
}

Received 8 December 2007 / Accepted 18 February 2008

\section{ABSTRACT}

Context. We present 31.2 days of nearly continuous MOST photometry of the rapidly oscillating Ap star 10 Aql.

Aims. The goal was to provide an unambiguous frequency identification for this little studied star, as well as to discuss the detected frequencies in the context of magnetic models and analyze the influence of the magnetic field on the pulsation.

Methods. Using traditional Fourier analysis techniques on three independent data reductions, intrinsic frequencies for the star are identified. Theoretical non-adiabatic axisymmetric modes influenced by a magnetic field having polar field strengths $B_{\mathrm{P}}=0-5 \mathrm{kG}$ were computed to compare the observations to theory.

Results. The high-precision data allow us to identify three definite intrinsic pulsation frequencies and two other candidate frequencies with low $S / N$. Considering the observed spacings, only one $(\Delta v=50.95 \mu \mathrm{Hz})$ is consistent with the main sequence nature of roAp stars. The comparison with theoretical models yields a best fit for a $1.95 M_{\odot}$ model having solar metallicity, suppressed envelope convection, and homogenous helium abundance. Furthermore, our analysis confirms the suspected slow rotation of the star and sets new lower limits to the rotation period $\left(P_{\text {rot }} \geq 1\right.$ month) and inclination $\left(i>30 \pm 10^{\circ}\right)$.

Conclusions. The observed frequency spectrum is not rich enough to unambiguously identify a model. On the other hand, the models hardly represent roAp stars in detail due to the approximations needed to describe the interactions of the magnetic field with stellar structure and pulsation. Consequently, errors in the model frequencies needed for the fitting procedure can only be estimated. Nevertheless, it is encouraging that models which suppress convection and include solar metallicity, in agreement with current concepts of roAp stars, fit the observations best.

Key words. techniques: photometric - stars: chemically peculiar - stars: individual: $10 \mathrm{Aql}$ - stars: individual: HD 176232 stars: magnetic fields - stars: oscillations

\section{Introduction}

Rapidly oscillating Ap (roAp) stars are classified as chemically peculiar main sequence stars pulsating with periods ranging from 6-21 $\mathrm{min}$. Since the first discovery by Kurtz (1978), more than 30 members have been identified and their pulsations are generally believed to be high-order low-degree magnetoacoustic modes. The roAp stars are characterized by strong global magnetic fields with polar strengths up to several $\mathrm{kG}$.

The discovery of a synchronous modulation of the pulsation amplitude with the magnetic field strength led to the oblique pulsator model, which assumes the pulsation axis is aligned with the magnetic axis which is itself oblique to the axis of rotation. This concept naturally explains the observed frequency triplets as axisymmetric dipolar modes modulated by the rotation period (Kurtz 1982). This model was challenged by Bigot \& Dziembowski (2002), who took into account rotational

* Based on data from the MOST satellite, a Canadian Space Agency mission, jointly operated by Dynacon Inc., the University of Toronto Institute for Aerospace Studies and the University of British Columbia, with the assistance of the University of Vienna. effects such as the centrifugal force and argued in favor of nonaxisymmetric modes. Assuming an axisymmetric mode aligned with the magnetic axis, Saio \& Gautschy (2004) found that the amplitude of the pulsation is confined to the magnetic axis, which was confirmed observationally by Kochukhov (2006) analyzing spectroscopic line profile variations of the rapidly rotating roAp star HR 3831.

The mechanism driving the pulsation in roAp stars is widely believed to be the $\kappa$-mechanism acting in the $\mathrm{H}$ ionization zone. Attempts have been made to explain the lack of low-order pulsation modes despite the vicinity to classical $\delta$ Scuti pulsators in the Hertzsprung-Russell diagram (HRD) by both indirect and direct effects of the magnetic field. With the inclusion of gravitational settling of helium in the outer layers of the star due to inhibited meridional circulation as well as suppression of envelope convection at the poles due to the magnetic field, it became possible to drive the high-order pulsations as observed (Balmforth et al. 2001); however, some lower order modes were also excited in these models.

Saio (2005) concentrated on the direct effect of the magnetic field, showing that the decoupling of the pulsation into an 
acoustic wave and a magnetic slow wave (which dissipates in the interior) is sufficient to damp out all the low-order modes, while high order modes remain unstable. Beside the excitation analysis, the direct effect on the observed frequencies has been intensively studied (e.g., Saio \& Gautschy 2004; Cunha 2006).

It has been shown that magnetic coupling in the layers where the magnetic pressure becomes comparable to the gas pressure can significantly alter the pulsation frequency. This, in turn, causes non-negligible deviations from the classical asymptotic theory (Tassoul 1980), which predicts equally spaced frequencies of consecutive radial overtones and the same degree and is widely used to interpret high-order p-modes for, e.g., solartype oscillations. It was shown that the observed deviations from these spacings depend essentially on the strength of the magnetic field as well as the field geometry, making accurate frequency determination a valuable tool in mode identification.

The rapid pulsation of $10 \mathrm{Aql}$ (HD 176232, HR 7167, $\mathrm{V}=$ 5.91) was first discovered by Heller \& Kramer (1990) using high speed photometry with the 1-m telescope of the Mount Laguna observatory. From about $30 \mathrm{~h}$ of observations, they were able to identify three pulsation frequencies at $f_{1, \mathrm{HK}}=1.4360 \mathrm{mHz}$, $f_{2, \mathrm{HK}}=1.3854 \mathrm{mHz}$ and $f_{3, \mathrm{HK}}=1.2393 \mathrm{mHz}$ all with amplitudes $<0.5 \mathrm{mmag}$. Due to severe aliasing in their data, and the low signal amplitudes, they noted that the identifications are not unambiguous; however, they estimated from the observed spacings that the large frequency separation (hereafter simply $\Delta v$ ) could be $50.6 \mu \mathrm{Hz}$. Shortly thereafter, Belmonte et al. (1991) published IR photometry which confirmed the detection by Heller \& Kramer (1990) with the signals in their amplitude spectra being close to the observed frequencies. Surprisingly, their observed amplitudes (obtained in the $J$ filter) were in some cases about a factor 2-3 greater than in the Johnson $B$ filter, and their highest peak was coincident with a $1 \mathrm{~d}^{-1}$ alias of $f_{3, \mathrm{HK}}$. One year later, Belmonte et al. (1992) returned to $10 \mathrm{Aql}$, but failed to redetect the signal by the same method.

IR photometry in the early 1990's constituted the last published photometric campaign of $10 \mathrm{Aql}$. Focusing on abundance analysis, Ryabchikova et al. (2000) obtained high resolution spectra of $10 \mathrm{Aql}$, estimating $T_{\text {eff }}$, radius and $\log g$ through synthetic line profile fitting. As for many other roAp stars, they detected a strong overabundance of the doubly ionized rare earth elements (REE's) Pr III and Nd III. Shortly thereafter, Kochukhov et al. (2002) succeeded in detecting the pulsation from radial velocity variations of these lines while also confirming the unusually low amplitude of the signal with peak amplitudes of $130-150 \mathrm{~m} \mathrm{~s}^{-1}$. They also provided the first measurement of the mean magnetic field modulus $\langle B\rangle=1.5 \pm 0.1 \mathrm{kG}$ from Zeeman splitting. Hatzes \& Mkrtichian (2005) confirmed the pulsation in the radial velocities for several lines. They also detected highly variable amplitudes from line to line, but also from night to night for the same line, which was interpreted as either rotational modulation, growth or decay of modes on short timescales, or close multiperiodicity. As the MOST observations will show, the latter explanation was most likely the reason.

In an effort to suggest $10 \mathrm{Aql}$ as a potential target for the space telescope COROT (Baglin \& The COROT Team 1998), Bigot \& Weiss (2002) published the first and, so far, last attempt to model the oscillation spectrum of this star. Assuming an effective temperature of $8000 \pm 100 \mathrm{~K}$ (based on photometric calibrations) and a luminosity of $21.4 \pm 0.6 L_{\odot}$ (obtained with the Hipparcos parallax), they found an agreement with the three frequencies published by Heller \& Kramer (1990) and the large frequency separation expected for a magnetic field strength of $0.8 \mathrm{kG}$.
Table 1. Basic data on the MOST observations of 10 Aql.

\begin{tabular}{cc}
\hline \hline Parameter & Value \\
\hline Obs. time [HJD-2 451 545] & $2371.08-2402.24$ \\
Total timespan [d] & 31.16 \\
Duty cycle [\%] & 98 \\
Exposure time [s] & 10 \\
Sampling time [s] & 20 \\
\hline
\end{tabular}

The rotation period of $10 \mathrm{Aql}$ is unknown; however, it is believed to be very long based on magnetic field measurements by Babcock (1958), which showed a reversal of polarity in a timespan of 4 years. Preston (1970) measured a constant mean longitudinal field strength of $\sim 500 \mathrm{G}$ over 10 consecutive nights. Ryabchikova et al. (2005) obtained 5 additional values over a timespan of one year, confirming the value measured by Preston some 30 years earlier. Considering the measured $v \sin i=2.0 \pm 0.5 \mathrm{~km} \mathrm{~s}^{-1}$ (Kochukhov et al. 2002) as an upper limit, they concluded that the rotational period might possibly be as long as hundreds of years. Since this, however, would contradict Babcock's observation and a pole-on configuration could not yet be excluded, there still was no securely measured lower limit for the rotation period of $10 \mathrm{Aql}$.

All spectroscopic campaigns conducted on $10 \mathrm{Aql}$ were based on observations spanning a couple of nights and were therefore unsuitable for confirming and precisely identifying the pulsation modes of the star. Although recent observations have shown the power of high resolution spectroscopy in probing the interior and even mapping the surface structure of pulsation in roAp stars (e.g., Kochukhov 2004), it is clear that a secure identification of all pulsation modes is indispensable for a complete understanding of the effects observed (in both photometry and spectroscopy). Due to the high precision and long uninterrupted time coverage, MOST is uniquely suited for such a campaign to perfectly complement recent accomplishments in the field of roAp stars.

\section{Photometry and data reduction}

The MOST (Microvariability and Oscillations of STars) space telescope (Walker et al. 2003) was launched in June 2003 to perform high precision uninterrupted photometry of bright stars. It is equipped with a Maksutov telescope with a $15 \mathrm{~cm}$ aperture, a custom broadband filter $(350-700 \mathrm{~nm})$ and, in its original design, two CCD's (one for science, the other for startracking). Due to its sun-synchronous, polar orbit with an orbital period of $\sim 101 \mathrm{~min}$, MOST is able to measure stars for up to two months without interruption, making it uniquely suited to obtain high precision frequency determinations free from aliasing effects. Early in 2006 the tracking CCD system failed due to a particle hit. Since then, both science and tracking have been carried out with the Science CCD system.

The star 10 Aql was observed by MOST from June 29July 30, 2006 as a primary science target. Because of its moderate brightness $(V=5.91)$ it was observed in the open field (corresponding to traditional CCD photometry, see Walker et al. 2003 , for details). A single exposure consisted of 20 added $0.5 \mathrm{~s}$ images for a total exposure time of $10 \mathrm{~s}$ at a sampling interval of 20 s. 119724 exposures were acquired with two interruptions due to difficulties with the satellite electronics, to yield a duty cycle of $98 \%$.

Due to parasitic stray light caused mostly by Earthshine scattered into MOST's focal plane, special data reduction techniques 
were developed to remove instrumental artifacts present in the raw data. In the case of $10 \mathrm{Aql}$, three different independent reduction techniques were applied. A newly developed reduction software developed for MOST open field photometry (Huber \& Reegen 2008) is based on a technique originally developed for Fabry imaging (Reegen et al. 2006), in which correlations of intensities of pixels illuminated by starlight and sky intensities are calculated over time. Performing a correction of a linear regression to the intensity-intensity diagrams, stray light effects are effectively eliminated from the data. To be able to apply this technique to open field photometry, the reduction applies an image shifting routine which aligns all images to common pixel coordinates using bilinear interpolation of sub-pixel intensities, allowing a definition of a fixed aperture over time independent of minor satellite jitter effects. The software allows a decorrelation of the mean as well as pixel intensities with the option of using higher order fits (depending on the complexity of the correlation). It additionally implements an identification and correction of pixels affected by cosmic ray hits as well as elimination of bad quality frames caused by pointing instabilities (see Huber \& Reegen 2008, for details).

An independent reduction using the open-field subraster was performed by JR and is described in detail by Rowe et al. (2006). This technique additionally implements flatfield and dark current corrections. Since there are no moveable parts on the satellite to obtain such calibration frames in the traditional sense, flatfield correction is performed by making use of the fact that during high stray light phases individual CCD subrasters are almost uniformly illuminated by scattered Earthshine. The software uses a combination of PSF fitting and aperture photometry to obtain the count rates of the star. Stray light influences are corrected similar to the reduction described above from a fit to mean star and sky intensities.

With the loss of the MOST startracking CCD another reduction capability was implemented. MOST acquires count rates for several guide stars, which are reduced on-board by simply subtracting a mean sky value after applying a given threshold. Since guide stars and science targets are on the same CCD, the guide star values are also returned for all science targets (in the case of $10 \mathrm{Aql}$, every $0.5 \mathrm{~s}$ ). The extracted on-board processed intensities were additionally reduced by $\mathrm{MH}$ by applying the decorrelation technique with other (constant) guide stars. While the accuracy of the count rate determination is certainly not of the same quality as in the actual subraster images, the big advantage of this reduction is that intensities are on-board reduced after each sub-exposure, avoiding possible smearing effects of stray light effects in stacked images.

All three reductions were performed independently and yielded different effective duty cycles (ranging from 63-95\%) depending on the outlier rejection philosophy used. Each reduction showed several strengths and weaknesses, and all three were used to secure the results obtained from the data. Fig. 1 shows the reduced light curve of $10 \mathrm{Aql}$, in which all signals with frequencies $<5 \mathrm{~d}^{-1}$ corresponding to instrumental trends have been removed. The data show an increased scatter towards the end of the run. Nevertheless, the bottom panel clearly shows the detection of roAp pulsation.

\section{Frequency analysis}

\subsection{Frequency identification}

Frequency analysis was performed using the programs SIGSPEC (Reegen 2007) as well as Period04 (Lenz \& Breger 2004).
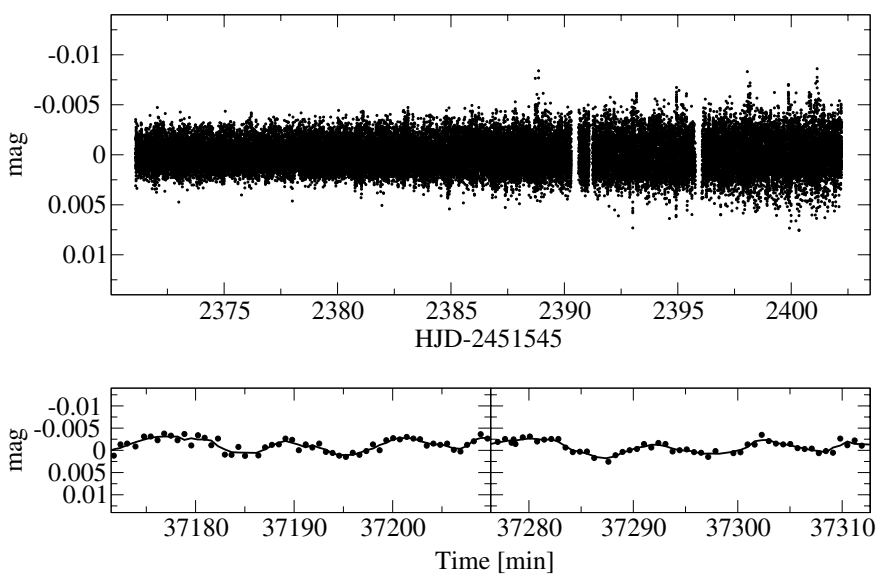

Fig. 1. Reduced light curve of 10 Aql. The bottom panels show a zoom of the light curve, at approximately HJD-2 451545 2396.9-2397.0 with a running average overplotted.

SIGSPEC computes a quantity called spectral significance based on a false-alarm probability by comparing the signal to white noise, and incorporating frequency, amplitude and phase information in the calculation. The program has proved to be extremely powerful for a statistically clean evaluation of the signal in Fourier space, and especially in the case of the high frequencies for the roAp stars where colored noise (intrinsic or instrumental) can be assumed to be negligible, the spectral significance gives important additional information compared to the more traditional $S / N$ estimate.

Figure 2 (top panel) shows a Fourier amplitude spectrum of the reduced $10 \mathrm{Aql}$ data in the frequency region of interest. With the dashed lines indicating the published frequency values of the two largest amplitude peaks by Heller \& Kramer (1990), the MOST data clearly show that indeed their frequencies were misidentified as $1 \mathrm{~d}^{-1}(=0.0116 \mathrm{mHz})$ aliases. In fact, for both cases the correct frequency lies $1 \mathrm{~d}^{-1}$ higher. Moreover, the MOST data enables us to resolve an additional, as of yet undetected, frequency around $1.427 \mathrm{mHz}$ at high $S / N$. Thanks to the clear spectral window showing only the aliasing effects spaced at MOST's orbital frequency $\left(14.19 \mathrm{~d}^{-1}=0.16 \mathrm{mHz}\right)$, the identification of these three frequencies is unambiguous. They appear consistently in all three reductions with comparable significances. To ensure the intrinsic nature of this signal, the middle panel of Fig. 2 shows the amplitude spectrum of the brightest star simultaneously observed (HD 175922, $V=7.21$ ) and additional peaks that were identified using the same approach with SIGSPEC to the light curve constructed with background readings of the $10 \mathrm{Aql}$ subraster. Clearly, no instrumental power can be identified in the vicinity of $f_{1}, f_{2}$ or $f_{3}$. We therefore definitely consider these frequencies to be the result of intrinsic stellar pulsation.

Apart from the three obvious peaks, $10 \mathrm{Aql}$ does not reveal any eye-catching power above the noise level of about $10 \mathrm{ppm}$. We do detect two peaks in the spectrum which were identified by SIGSPEC with a spectral significance of 6 , corresponding roughly to a $S / N \geq 4$. These frequencies are marked in Fig. 2 by the labels $f_{i}$ and $f_{j}$. Unfortunately, these peaks are only identified in the reduction showing the lowest noise level in this region. The comparison data (middle panel, Fig. 2) does not reveal any obvious power in this frequency region; however, one must note that the amplitudes of $f_{i}$ and $f_{j}$ are at about the noise level of that in the fainter comparison star. Clearly, we are not able to infer from the data available if these peaks are indeed intrinsic 


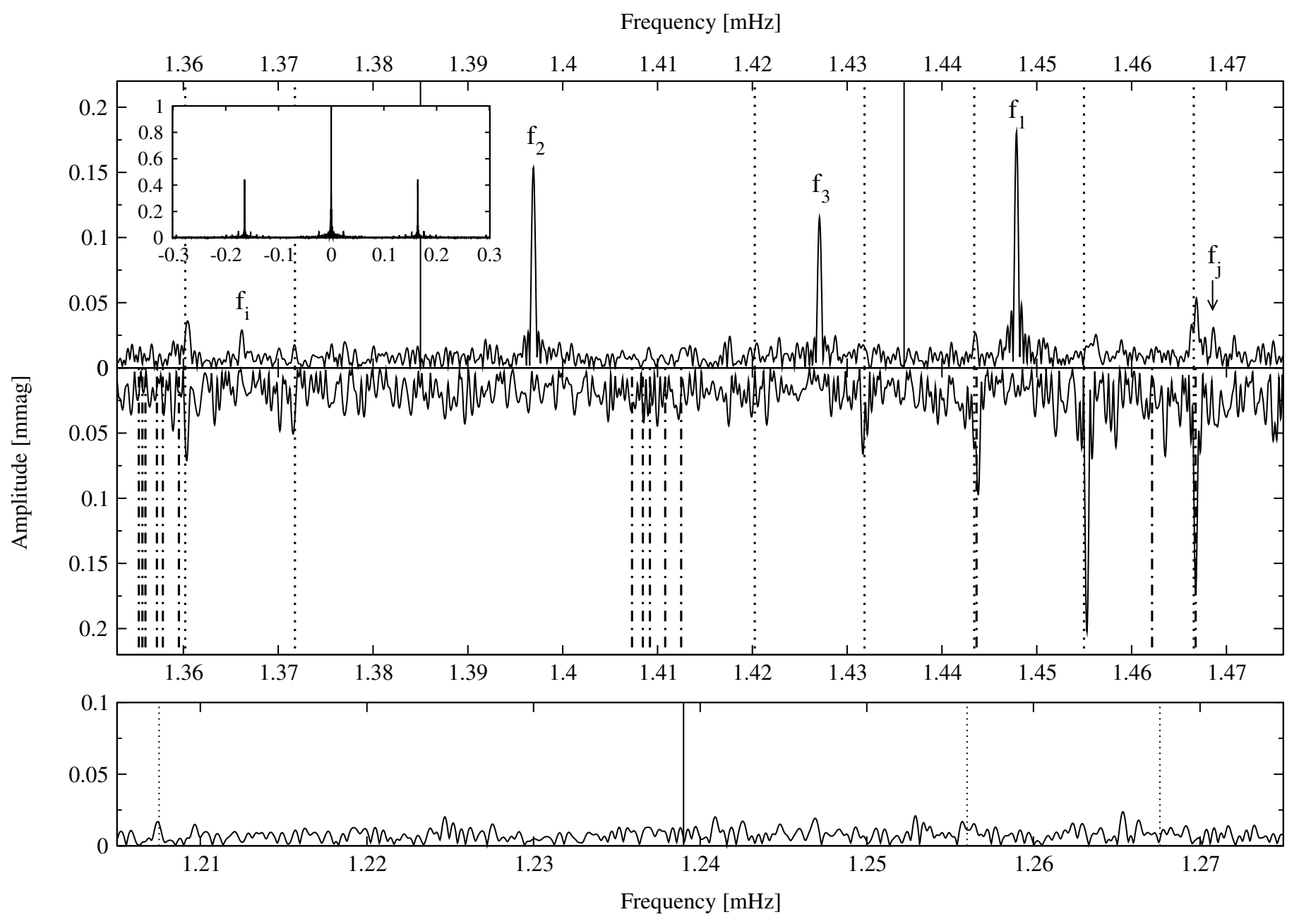

Fig. 2. (Top panel) Amplitude spectrum of 10 Aql. Solid lines indicate the position of published frequencies by Heller \& Kramer (1990). Dotted lines show the position of $1 \mathrm{~d}^{-1}$ sidelobes of harmonics of MOST's orbital frequency (i.e., instrumental artifacts). The insert displays the spectral window. (Middle panel) Inverted amplitude spectrum of the brightest guide star simultaneously observed (HD 175922, $V=7.21$ ), shown at the same scale. Dashed-dotted lines indicate the position of frequencies identified in the background readings of the 10 Aql subraster. (Bottom panel) Frequency region where $f_{3, \mathrm{HK}}$ was reported after prewhitening of $f_{1}, f_{2}$ and $f_{3}$ (the position again indicated by a solid line).

or not. Nevertheless, we consider them as candidate frequencies and included them in the subsequent analysis.

Finally, the bottom panel of Fig. 2 shows the frequency region where an additional mode has been reported by Heller \& Kramer (1990) and Belmonte et al. (1991). We do not detect any significant power at $f_{3, \mathrm{HK}}$ or any surrounding $1 \mathrm{~d}^{-1}$ aliases thereof (as suggested by Belmonte et al. 1991). Considering that $f_{3 \text {,HK }}$ was the lowest amplitude frequency in the dataset of Heller \& Kramer (1990), one might conclude from this result that their detection was indeed an instrumental artifact. Nevertheless, the fact that Belmonte et al. (1991) also found a signal at almost exactly the same value (in their case, the highest amplitude peak) makes this explanation more unlikely. On the other hand, their follow-up observations one year later did not show evidence for any detectable signal at all using the same methods (Belmonte et al. 1992).

If the detections were real, we could conclude that the lack of signal in the MOST data is in fact evidence for a limited mode lifetime on the order of some decades, possibly caused by damping of the mode by intrinsic changes in the star, or a different orientation of the star at the time of MOST observations due to rotation over the past 15 years. Due to the uncertainty of both earlier detections, however, these conclusions must remain in the realm of speculation at this point.

Apart from the frequency region just discussed, the $10 \mathrm{Aql}$ data do not reveal any variability at lower (or higher) frequencies with a $S / N \geq 4$ which cannot be attributed to instrumental artifacts. This is consistent with previously published results demonstrating $10 \mathrm{Aql}$ to be a slow rotator (i.e., no frequency multiplets due to rotational modulation are observed) and theoretical predictions that no low-order p-modes are excited in roAp stars. We are, furthermore, not able to detect harmonics of any definite frequencies $f_{1}, f_{2}$ or $f_{3}$, contrary to what has been found for the MOST observations of $\gamma$ Equ (Gruberbauer et al. 2008). Table 2 lists all identified intrinsic and candidate frequencies in the MOST $10 \mathrm{Aql}$ dataset. Note that the periods of the stated frequencies correspond to 11.3-12.2 min and that errors of the frequency, amplitude and phase values have been computed incorporating the spectral significance as described in Kallinger et al. (2008).

\subsection{Amplitude modulation}

Apart from mean light variations which are believed to be due to abundance spots on the surface of Ap stars, amplitude 
Table 2. List of frequencies identified in the MOST 10 Aql observations. Candidate frequencies are denoted $f_{i}$ and $f_{j}$. The phase values shown are calculated as $\sin (2 \pi(f t+\theta))$ with the zero point corresponding to JD 2451545.

\begin{tabular}{ccccc}
\hline \hline Id & $f[\mathrm{mHz}]$ & $A[\mathrm{mmag}]$ & $\theta[\mathrm{rad}]$ & $\mathrm{sig}$ \\
\hline$f_{1}$ & $1.44786 \pm 0.00003$ & $0.17 \pm 0.01$ & $0.2 \pm 0.3$ & 135 \\
$f_{2}$ & $1.39691 \pm 0.00003$ & $0.15 \pm 0.01$ & $0.8 \pm 0.3$ & 101 \\
$f_{3}$ & $1.42709 \pm 0.00004$ & $0.12 \pm 0.01$ & $0.7 \pm 0.4$ & 68 \\
$f_{i}$ & $1.3662 \pm 0.0001$ & $0.03 \pm 0.02$ & $0.3 \pm 1.2$ & 6 \\
$f_{j}$ & $1.4686 \pm 0.0001$ & $0.03 \pm 0.02$ & $0.9 \pm 1.2$ & 6 \\
\hline
\end{tabular}

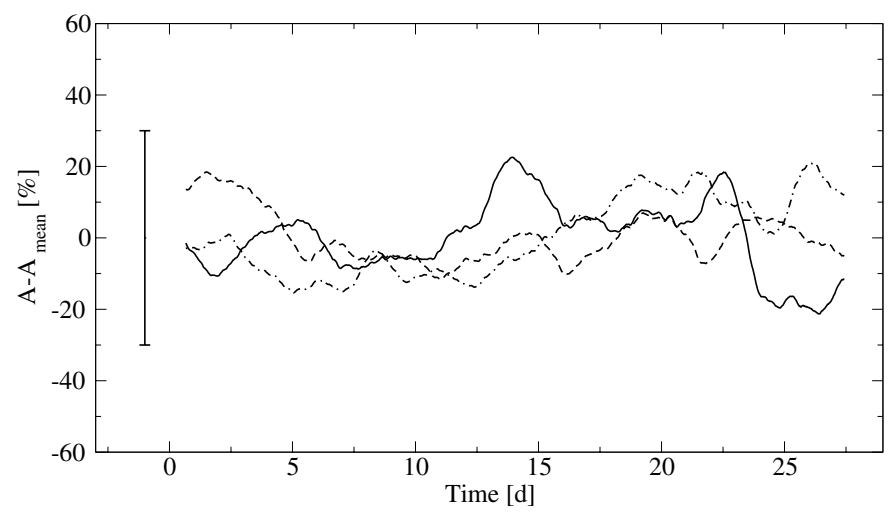

Fig. 3. Amplitude modulation of $f_{1}$ as determined by time resolved frequency analysis. The different lines correspond to the three reductions performed for the dataset (solid: Direct Imaging (Vienna); dashed: Direct Imaging (UBC); dashed-dotted: Guide Star), and each line shows a smoothed curve of the individual values. The ordinate is the relative amplitude change. The error bar indicates the mean error for all three reductions for a single datapoint.

modulation of an intrinsic frequency might present clues about the rotation period. Following the oblique pulsator model, the magnetic poles (and hence the pulsation poles) would wander in and out of sight as the star rotates, modulating the pulsation amplitude with exactly that period. As the 31.2 days of MOST observations constitute the longest continuous dataset ever obtained for $10 \mathrm{Aql}$, we are able to set a new limit to the so far unknown rotation period of this star.

The modulation was investigated by time resolved frequency analysis using SigSPEc. Subsets were chosen long enough to resolve the frequency component with a secure detection limit (in this case $3.2 \mathrm{~d}$ ), with stepwidths equal to one pulsation period. This exercise was performed for all three intrinsic frequencies and all three independent reductions. The results for $f_{1}$ are shown in Fig. 3. While the amplitude does change up to $25 \%$ throughout the run, none of the variations are consistent within the different reductions. Similar results have been obtained for $f_{2}$ and $f_{3}$. We conclude that the variations seen are purely due to different data and reduction qualities at different observation times, and that no significant amplitude modulation is present in the $10 \mathrm{Aql}$ data. Considering the timespan of 31.2 days of observations, we can therefore rule out that the star rotates with a period shorter than about a month (assuming that the star is not seen pole-on, in which case no modulation would be detected even for a rapidly rotating star). This conclusion is also confirmed by time-resolved spectroscopy which was obtained simultaneously to the MOST photometry spanning over 21 days, yielding constant line intensities within $0.5 \%$ (Sachkov et al. 2008). Both results are consistent with earlier observations that
10 Aql is a slow rotator and set a new lower limit for its rotation period.

\section{Asteroseismic analysis}

\subsection{Frequency spacings}

A fundamental observational parameter in stars pulsating with high-order acoustic modes is the large frequency separation $\Delta v$, describing the regular spacing of consecutive radial overtones $n$ with same degree $\ell$. Since it is proportional to the square root of the mean density of the star, it allows an estimation of astrophysical parameters such as luminosity and effective temperature.

Obviously, the intrinsic frequencies observed in $10 \mathrm{Aql}$ do not reveal a regular spacing. The observed spacings are as follows:

$f_{1}-f_{2}=\Delta_{1}=50.95 \pm 0.03 \mu \mathrm{Hz}$
$f_{3}-f_{2}=\Delta_{2}=30.19 \pm 0.03 \mu \mathrm{Hz}$
$f_{1}-f_{3}=\Delta_{3}=20.76 \pm 0.03 \mu \mathrm{Hz}$

Considering roAp theory as mentioned in the introduction, this result is not surprising, since it has been shown that the magnetic field can significantly perturb the observed frequencies and therefore the spacing. On the other hand, it might be possible that what we observe are in fact modes of different degree $\ell$. It is, however, interesting to note that $f_{2}-f_{i} \sim \Delta_{2}$ as well as $f_{j}-f_{1} \sim \Delta_{3}$. This might give rise to the assumption that either $f_{1}$ or $f_{2}$ are perturbed by the magnetic field, which, if those values were slightly shifted, give four consecutive frequencies spaced by either $\Delta_{2}$ or $\Delta_{3}$.

To test these speculations, we used the published HRD (Hertzsprung Russell Diagram) position by Matthews et al. (1999) as well as Kochukhov \& Bagnulo (2006). They calculate the luminosity based on the measured Hipparcos parallax and derive an effective temperature based on photometric calibrations. The frequency spacing, $\Delta v$, is directly connected to physical properties which determine the position of the star in the HRD, as given for example by Matthews et al. (1999) via

$\Delta v=(6.64 \pm 0.36) \times 10^{-16} M^{0.5} T_{\mathrm{eff}}^{3} L^{-0.75} \mathrm{~Hz}$,

where $M$ is given in solar masses, $L$ in solar luminosities and $T_{\text {eff }}$ in Kelvin. Hence, it is possible to identify models showing a constant $\Delta v$ and compare them with the previously determined values. The result is shown in Fig. 4. In this theoretical HRD, evolutionary tracks using the Yale Stellar Evolution Code (Guenther et al. 1992) are plotted, together with lines of constant $\Delta v$ of 50 and $30 \mu \mathrm{Hz}$, respectively.

Clearly, any large frequency separation considerably less than $50 \mu \mathrm{Hz}$ would contradict (for the given temperature and luminosity region) the current understanding of roAp stars as objects between ZAMS and TAMS. Note that although these evolutionary tracks do not take into account any effects of the magnetic field, frequency jumps on the order of several tenths of $\mu \mathrm{Hz}$ are expected to occur only at discrete frequency values rather than constantly for each frequency (Cunha 2006). More likely, consecutive shifts are on the order of a few $\mu \mathrm{Hz}$. It is therefore not possible that the magnetic field perturbations mimic a $\Delta v$ which is different from that expected from the physical parameters of the star. In other words, Eq. (1) is still a valid approximation even for modes perturbed by a magnetic field, except for large jumps at certain overtones. It is interesting to note that this 


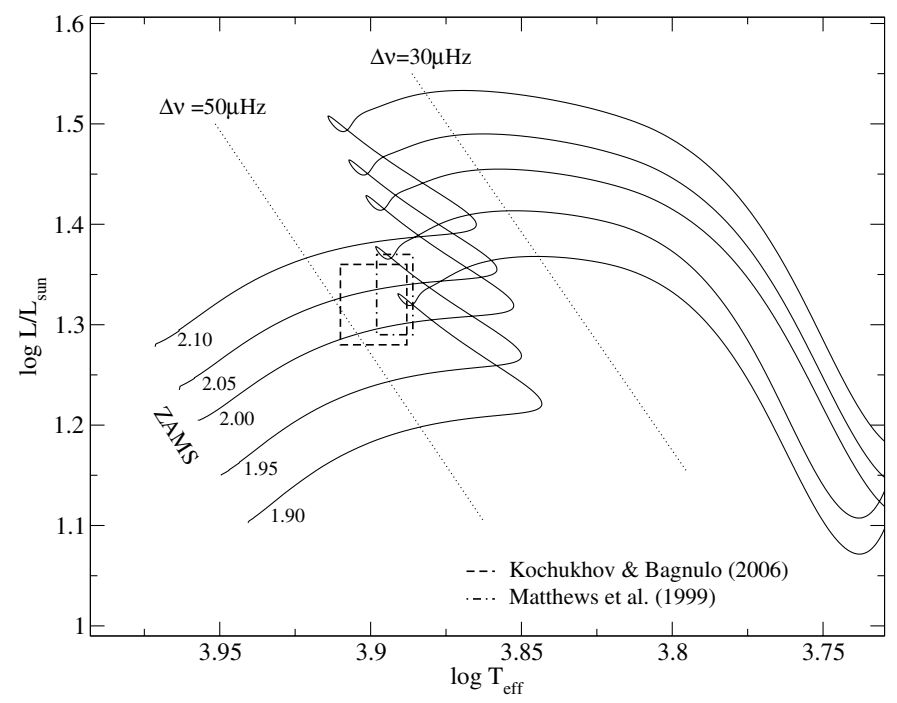

Fig. 4. Theoretical HRD showing evolutionary tracks with $(X, Z)=$ $(0.71,0.19)$ and $\alpha=1.8$ (Guenther et al. 1992). Numbers next to the tracks show the mass in solar units. The dotted lines indicate contours of constant $\Delta v$. Previously determined parameters for $10 \mathrm{Aql}$ are shown.

Table 3. Summary of models used for the analysis.

\begin{tabular}{ccccc}
\hline \hline Id & $M / M_{\odot}$ & $(X, Z)$ & He depl & Conv \\
\hline S190 & 1.90 & $(0.700,0.020)$ & yes & no \\
S195 & 1.95 & $(0.700,0.020)$ & yes & no \\
S200 & 2.00 & $(0.700,0.020)$ & yes & no \\
S205 & 2.05 & $(0.700,0.020)$ & yes & no \\
\hline H195 & 1.95 & $(0.700,0.020)$ & no & no \\
H200 & 2.00 & $(0.700,0.020)$ & no & no \\
H205 & 2.05 & $(0.700,0.020)$ & no & no \\
\hline M200 & 2.00 & $(0.695,0.025)$ & yes & no \\
\hline C195 & 1.95 & $(0.700,0.020)$ & no & yes \\
\hline
\end{tabular}

value for $\Delta v$ was actually already suggested after the first photometric campaign by Heller \& Kramer (1990). Although less accurate and based on wrong frequency identifications, their first asteroseismological interpretation of $10 \mathrm{Aql} \mathrm{might} \mathrm{turn} \mathrm{out} \mathrm{to} \mathrm{be}$ correct after all.

\subsection{Model frequencies}

To further quantify the observations of $10 \mathrm{Aql}$, we performed a comparison with models which were calculated from the zeroage main sequence to the end of the main sequence for masses ranging from $1.90-2.05 M_{\odot}$ in steps of $0.05 M_{\odot}$ using OPAL opacity tables (Iglesias \& Rogers 1996). In standard models, an initial chemical composition $(X, Z)=(0.70,0.02)$ is adopted and envelope convection is suppressed. Helium abundance is taken as $Y=0.01+0.27\left(x_{2}+x_{3}\right)$, where $Y$ is the helium mass fraction and $x_{2}$ and $x_{3}$ represent fractions of singly and doubly ionized helium, respectively. For standard models, helium is depleted at the surface $\left(Y_{\mathrm{S}}=0.01\right)$ due to gravitational settling in the outermost layers of the star. Additionally, some non-standard models were computed which included metal-rich composition $(Z=0.025)$, envelope convection or assumed no helium depletion near the surface $\left(Y_{\mathrm{S}}=0.28\right)$. Table 3 lists all the stellar models used in our analysis.

Theoretical non-adiabatic frequencies of axisymmetric $(m=$ 0 ) high-order p-modes under the influence of a dipole magnetic field were calculated using the method described in Saio (2005).
Outer boundary conditions are imposed at an optical depth of $10^{-3}$. A reflective mechanical condition $(\delta p / p \rightarrow$ const.) was used although for models with $Y_{\mathrm{S}}=0.01$, the acoustic cut-off frequency is close to the frequencies observed for $10 \mathrm{Aql}$ (for models without helium depletion the critical frequency is higher than the observed frequencies, therefore justifying the use of a reflective boundary condition). Due to the neglect of possible kinetic energy leakage at the outer boundary, and the use of a diffusion approximation for radiative energy transport, the prediction for the stability of the modes in these models may not be very reliable.

Eigenfunctions of theoretical frequencies are expanded in terms proportional to spherical harmonics $\mathrm{Y}_{\ell}^{m=0}(\theta, \phi)$. The character of the latitudinal amplitude variation is designated by $\ell_{\mathrm{B}}$, the $\ell$ value of the expansion component having the largest kinetic energy. The value of $\ell_{\mathrm{B}}$ can change as the eigenfunction of a mode is modified by the effects of the magnetic field. In fact, a $\ell_{\mathrm{B}}=0$ mode sometimes changes to a $\ell_{\mathrm{B}}=2$ mode or vice versa. Generally, frequencies of $\ell_{\mathrm{B}}=0,3$ modes are strongly affected by a magnetic field, while $\ell_{\mathrm{B}}=1,2$ modes tend to be less affected. The latter modes can therefore be used to determine the position in the HR diagram and the former modes to estimate the strength of the magnetic field.

Theoretical frequencies were calculated for the HRD position estimated by previous observations as well as the observed frequency spacings and for magnetic field influences ranging from $B_{\mathrm{P}}=0-5 \mathrm{kG}$.

Because of the necessary simplifications, the models are far from representative of roAp stars in detail. For example, the magnetic field is assumed to be a simple centered dipole. Consequently, errors of model frequencies needed for the comparison with observational results can only be estimated. An additional severe restriction in the models is that only axisymmetric modes are considered. In the case of $10 \mathrm{Aql}$, however, this assumption can be justified by the fact that it has been repeatedly shown that this star has a very long rotation period possibly in the order of years, in which case rotationally perturbed modes are negligible. As has been shown in Sect. 3.2, this is also supported by MOST photometry.

\subsection{Model fitting}

Due to the fact that the calculation of model frequencies is very time consuming, the resolution of the original grid of models was enhanced by linear interpolation as described by Gruberbauer et al. (2008) in the application to the MOST observations of $\gamma$ Equ. The stepsize was decreased to $0.02 \mathrm{kG}$ in $B_{\mathrm{P}}$ as well as 0.00001 in $\log L / L_{\odot}$ and $\log T_{\text {eff }}$.

Observed frequencies were compared to theoretical ones using the $\chi^{2}$ statistics test as described by Guenther \& Brown (2004) and defined as

$$
\chi^{2}=\frac{1}{N} \sum_{i=1}^{N} \frac{\left(v_{\mathrm{obs}, i}-v_{\mathrm{mod}, i}\right)^{2}}{\sigma_{\mathrm{obs}, i}^{2}+\sigma_{\mathrm{mod}, i}^{2}},
$$

where $v_{\mathrm{obs}, i}$ and $v_{\text {model }, i}$ are the observed and corresponding model frequency, and $\sigma_{\mathrm{obs}, i}$ and $\sigma_{\text {mod, } i}$ are the uncertainties of these values, respectively. $N$ denotes the total number of frequencies fitted. For the uncertainty of model frequencies a value of $0.2 \mu \mathrm{Hz}$ was assumed. Using this formalism, a set of observed frequencies is considered to match the theoretical frequencies within the errors for values of $\chi^{2} \leq 1$. We note that with this formulation we do not draw any conclusions about over- or underestimation of errors (e.g., a reduced $\chi^{2} \ll 1$ value would, 

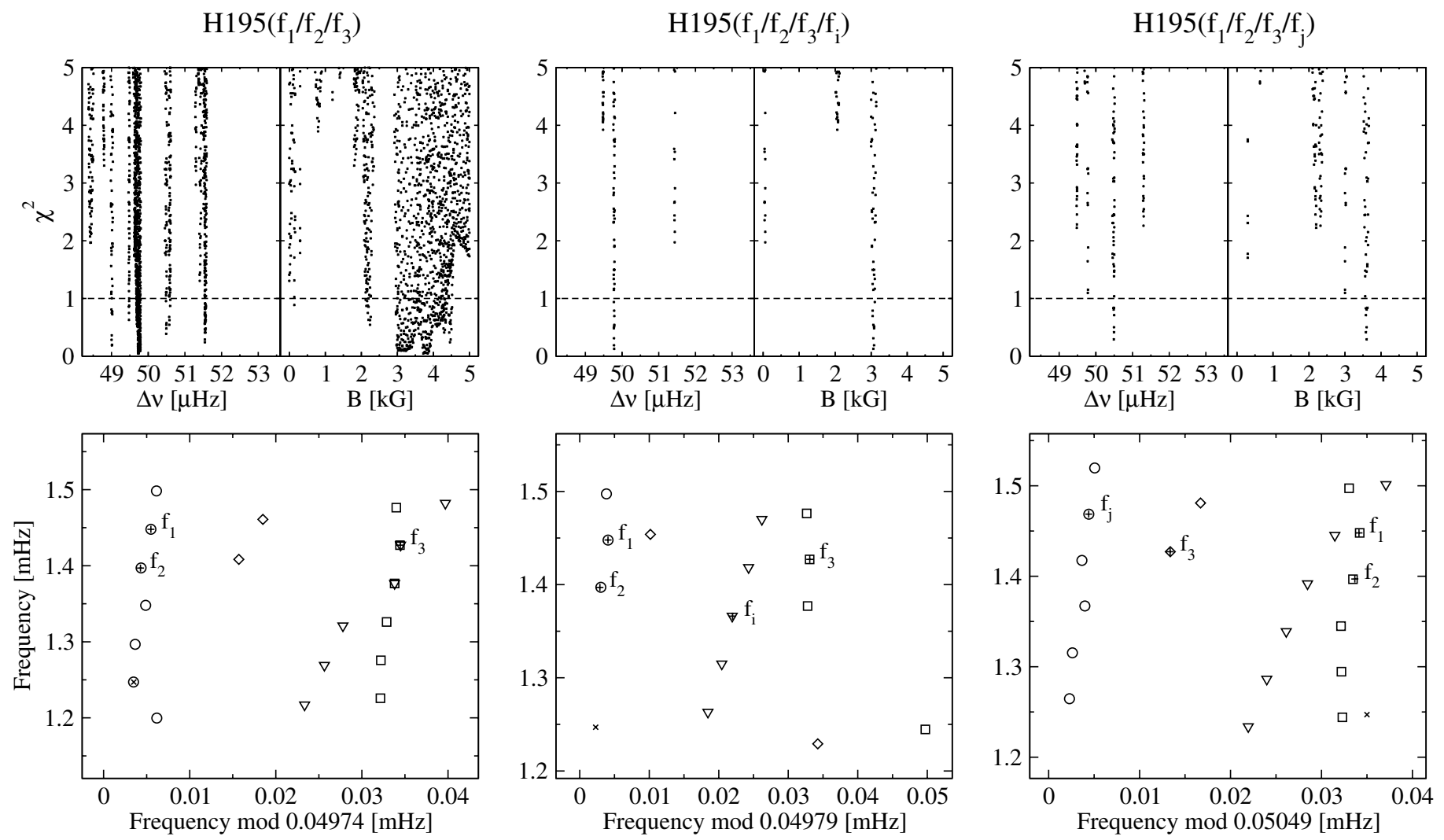

Fig. 5. Graphical presentation of the model fitting results for different frequency combinations in the H195 model. Top panels: $\chi^{2}$ values as a function of the parameter space covered by the models in $\Delta v$ (i.e., mass, effective temperature and luminosity) and $B_{\mathrm{P}}$, respectively. Bottom panels: Echelle diagrams of models with the lowest $\chi^{2}$. Spherical degrees $\ell_{\mathrm{B}}=0$ (triangles), $\ell_{\mathrm{B}}=1$ (squares), $\ell_{\mathrm{B}}=2$ (circles) and $\ell_{\mathrm{B}}=3$ (diamonds) are displayed, with observed MOST frequencies labeled and marked by plus signs. The cross in each plot shows the position of a frequency value published by Belmonte et al. (1991) at $1.247 \mathrm{mHz}$. Both errors for observed frequencies and theoretical frequencies are slightly smaller than the symbol sizes.

Table 4. Model fitting results for each combination of definite and candidate frequency set. Bold numbers show the best fitting results for a combination.

\begin{tabular}{ccccc}
\hline \hline $\begin{array}{c}\text { Model } \\
\text { id }\end{array}$ & $\left(f_{1} / f_{2} / f_{3}\right)$ & $\left(f_{1} / f_{2} / f_{3} / f_{i}\right)$ & $\begin{array}{c}\chi^{2} \\
\left(f_{1} / f_{2} / f_{3} / f_{j}\right)\end{array}$ & $\left(f_{1} / f_{2} / f_{3} / f_{i} / f_{j}\right)$ \\
\hline S190 & 0.27 & 1.51 & 0.94 & 6.35 \\
S195 & 0.13 & 0.55 & 1.33 & 7.96 \\
S200 & 0.10 & 0.38 & 0.69 & $\mathbf{2 . 1 0}$ \\
S205 & 0.26 & 0.46 & 2.63 & 2.61 \\
H195 & 0.05 & $\mathbf{0 . 1 3}$ & $\mathbf{0 . 2 9}$ & 4.54 \\
H200 & $\mathbf{0 . 0 2}$ & 2.62 & 2.05 & 3.50 \\
H205 & 0.13 & 1.40 & 4.71 & 4.08 \\
C195 & 0.11 & 0.84 & 2.33 & 8.32 \\
M200 & 0.30 & 5.20 & 4.33 & 15.2 \\
\hline
\end{tabular}

in the strictly statistical sense, indicate an overestimation of errors), but rather solely use the relative $\chi^{2}$ values to differentiate between the quality of the fits.

Since the intrinsic nature of the frequencies $f_{i}$ and $f_{j}$ cannot be confirmed beyond doubt, model fitting was performed with all combinations of definite and candidate frequencies. The results are summarized in Table 4. As can be seen from the $\chi^{2}$ values, the observed frequencies fit the theoretical ones well, if not to say too well considering the rather crude magnetic stellar models. Interestingly, when using all five frequencies, no fits within the errors can be found. This naturally leads to the conclusion that, assuming the theoretical frequencies are correct, either $f_{i}$ or $f_{j}$ must not be intrinsic to the star. On the other hand, if only the definite frequencies are used, all models fit within the errors, showing that an additional candidate frequency is needed to further constrain the models. Therefore, the focus of attention was brought to the combinations including only one of the two candidate frequencies.

Although the diversity of $\chi^{2}$ values for all models is quite weak, one can see that (apart from the combination using only definite frequencies), some non-standard models seem to clearly yield worse fits. This is true for models including envelope convection as well as models with higher metallicity $Z$. The former result is consistent with roAp theory, showing that suppressed envelope convection is a necessary condition for driving pulsation in these stars (e.g., Balmforth et al. 2001; Saio 2005) as has also been found by Gruberbauer et al. (2008) for the MOST observations of $\gamma$ Equ.

Considering only frequency combinations where fits with $\chi^{2}<1$ can be found, it can be seen that the best fits consistently occur for models without helium depletion. Examining the best fit for the set of definite frequencies (H200), it was found that the corresponding polar magnetic field value lies at $0.7 \mathrm{kG}$. While this value seems to be in agreement with the values derived by Bigot \& Weiss (2002) and Kochukhov et al. (2002), it must be noted that these authors considered the mean magnetic field strength, whereas the pulsation models test the polar magnetic field, which is expected to be considerably higher. Therefore, this result would stand in direct contradiction to the observed value inferred by Kochukhov et al. (2002). Furthermore, the two primary frequencies are identified as radial $\left(\ell_{\mathrm{B}}=0\right)$ modes in 
this model, which contradicts evidence found hitherto for roAp stars as being non-radial pulsators at least for large amplitude frequencies. For these reasons, we conclude that also for the combination of $f_{1} / f_{2} / f_{3}$ the H195 model, yielding a higher $\chi^{2}$ value (but still $\leq 1$ ) and a polar magnetic field value of $\sim 3.7 \mathrm{kG}$, presents a more reasonable solution.

In the top panels of Fig. 5, $\chi^{2}$ is shown as a function of $\Delta v$ and $B_{\mathrm{P}}$ for the $\mathrm{H} 195$ model. To present the results more easily, $T_{\text {eff }}, L$ and $M$ have been combined as $\Delta v$ according to Eq. (1). Including $f_{i}$, the regions showing low $\chi^{2}$ reduce dramatically (as expected), with only one region in $\Delta v$ and $B_{\mathrm{P}}$ having $\chi^{2}<1$. The same is valid for the combination including $f_{j}$, which, however, does not restrict the parameter space as much as the former combination, with the best fit lying at a slightly higher $B_{\mathrm{P}}$ and higher $\Delta v$.

As shown by Saio \& Gautschy (2004) and Saio (2005), there are certain values of $B_{\mathrm{P}}$ where the damping of acoustic waves due to magnetic slow waves reaches its maximum. In some cases the expansion of eigenfunctions used in the method does not converge, resulting in gaps in the theoretical frequencies. Note that the region between $B_{\mathrm{P}}=2.3-3 \mathrm{kG}$ shows such a gap for our set of frequencies.

In addition to the frequencies observed by MOST, we test the values provided by Heller \& Kramer (1990) and Belmonte et al. (1991) for the possible (but undetected in MOST data) additional frequency around $1.24 \mathrm{mHz}$. Indeed, one of the three values published by Belmonte et al. (1991) (1.247 $\mathrm{mHz}$ ) would fit the same sequence of $f_{1}$ and $f_{2}$, as shown in Fig. 5 by the cross in the corresponding Echelle diagram. Note that this is not the case for the combinations with $f_{j}$ and that for the combination using $f_{i}$, fewer theoretical modes are present since the fit lies very close to the critical $B_{\mathrm{P}}$ introducing a gap in the parameter space. Although this result is encouraging, we do not feel comfortable basing a model fit on a frequency that is not observed by MOST. Nevertheless, this is another indication that $10 \mathrm{Aql}$ indeed might have pulsated with an additional, slightly longer period in the early 1990's.

It might appear questionable to prefer the quality of one fit over the other when both show a $\chi^{2}<1$, i.e. both solutions fit within the errors. We argue that this is reasonable with the following example: the calculation of the minimum mean deviation of observed to theoretical frequencies, which does not incorporate any error values, gives (e.g., for the combination of certain frequencies) a value of $0.03 \mu \mathrm{Hz}$ for the $\mathrm{H} 195$ model. The corresponding standard model S195 (also showing a $\chi^{2}<1$ close to the H195 model, see Table 4) yields a mean deviation of $0.07 \mu \mathrm{Hz}$, meaning that the expected values of both theoretical and observed models are closer together for the model showing the lower $\chi^{2}$ value. If we now assume that the error distribution is Gaussian, it is clear that fits with a lower mean deviation from the expected values imply a higher probability that the solution is in agreement with the real (unknown) values.

Despite of this argument, it is clear from the results that there are not enough definite frequencies in the MOST $10 \mathrm{Aql}$ dataset to obtain a unique solution. Also, it is not possible to clearly rule out whether $f_{i}$ and $f_{j}$ are artifacts or not. Indeed, tests have shown that if random values or frequencies detected in simultaneously observed stars with a comparable significance to $f_{i}$ or $f_{j}$ are added to the set of definite frequencies, in about $50 \%$ of the cases a fit showing a $\chi^{2}<1$ can be found for the H195 model. This result demonstrates the fact that such a small quantity of observed frequencies does not allow one to derive unambiguous results for this star and all conclusions based on these fits should be treated with caution. Nevertheless, it can be concluded from

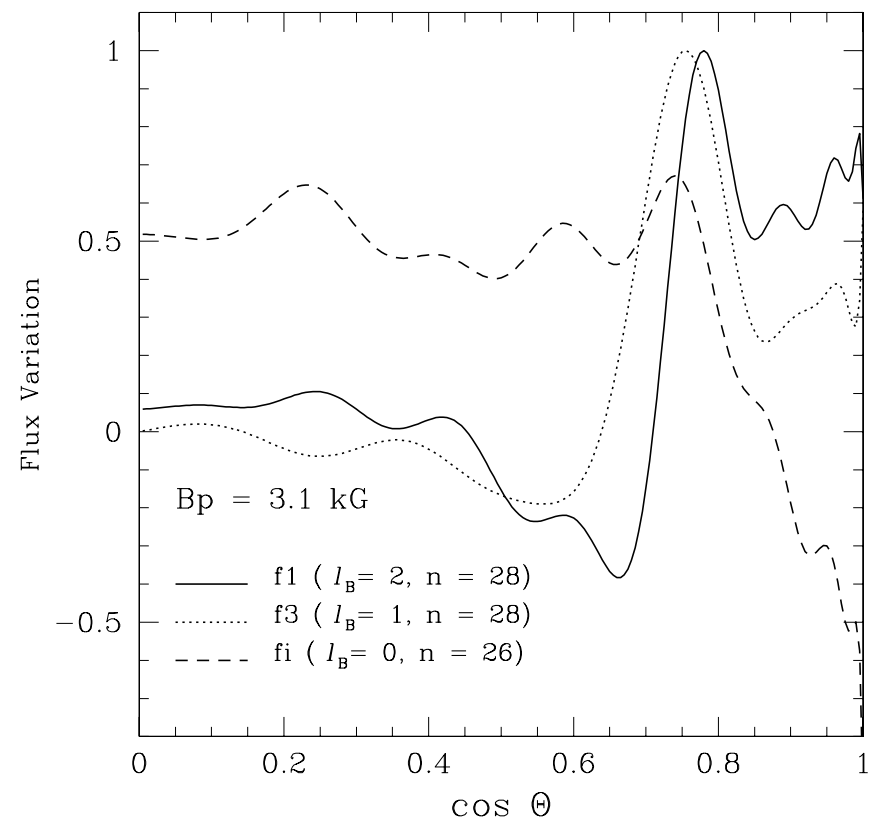

Fig. 6. Flux perturbation as a function of co-latitude $\Theta(\cos \Theta=1$ referring to the poles) for the best fitting non-interpolated H195 model $\left(\log T_{\text {eff }}=3.8883, \log L / L_{\odot}=1.2473\right)$. Note that $f_{2}\left(\ell_{\mathrm{B}}=2, n=27\right)$ is not specifically shown since the variation coincides with $f_{1}$.

the attempts presented here that the most reasonable fits seem to occur consistently for one stellar model, and that the results including $f_{i}$ seem to constrain the parameter space better for this model.

The bottom panel of Fig. 5 shows the Echelle diagrams of the models with the lowest $\chi^{2}$, together with the observed frequencies and a mode identification. Clearly, it is quite dangerous to derive a mode identification considering the low $\chi^{2}$ values given in Table 4 for other models. Nevertheless, we note that for the best solution including $f_{\mathrm{i}}$ the mode identification $\left(\ell_{\mathrm{B}} / n\right)$ would be as follows: $f_{1}=(2,28), f_{2}=(2,27), f_{3}=(1,28)$ and $f_{\mathrm{i}}=(0,26)$. If $f_{\mathrm{j}}$ is included, the modes would be identified as $f_{1}=(1,28), f_{2}=(1,27), f_{3}=(3,27)$ and $f_{\mathrm{j}}=(2,28)$. Figure 6 shows, for the closest non-interpolated model to our best fit, the flux perturbation as a function of the co-latitute $\Theta$ at the phase of maximum light for each mode. It can be seen that for all modes maximum light is concentrated towards $\Theta \sim 0.7$, and that the radial mode exhibits larger amplitudes towards the equator.

\subsection{Comparison with previous results}

Although the results of fitting are not unambiguous, we can compare our best fitting model with previously determined parameters derived using non-asteroseismological observables. The result of this comparison is shown in a theoretical HRD in Fig. 7. In addition to the results derived by Matthews et al. (1999) and Kochukhov \& Bagnulo (2006), we plot the position of our best model fit using the combinations with $f_{i}$ (filled diamond; note that the combination with $f_{j}$ only slightly changes this position) as well as an estimate using the observed large frequency spacing in combination with non-asteroseismological parameters by applying Eq. (1). The most reliable effective temperature estimation for $10 \mathrm{Aql}$ is from Ryabchikova et al. (2000) who fitted line profiles to the spectral energy distribution, yielding $T_{\text {eff }}=7550 \pm 150 \mathrm{~K}$. The mass was assumed to be $1.95 \pm$ $0.05 M_{\odot}$. Using these combined parameters (partially) based on 
Table 5. Astrophysical parameters derived for 10 Aql based on calculations using the observed spacing and model fits (using $f_{i}$ ). Bold face numbers show parameters based on estimations and non-asteroseismic observables, respectively. Errors for the model fit have been estimated by considering only grid points fits with $\chi^{2}<1$. The model mass is a fixed parameter.

\begin{tabular}{ccc}
\hline \hline Parameter & Using $\Delta v_{\text {obs }}$ & Model fit \\
\hline$M / M_{\odot}$ & $\mathbf{1 . 9 5} \pm \mathbf{0 . 0 5}$ & 1.95 \\
$T_{\text {eff }}$ & $\mathbf{7 5 5 0} \pm \mathbf{1 5 0}$ & $7734.5 \pm 0.5$ \\
$L / L_{\odot}$ & $15.6 \pm 1.7$ & $17.6885 \pm 0.0005$ \\
$B_{\mathrm{P}}[\mathrm{kG}]$ & - & $3.07 \pm 0.03$ \\
$R / R_{\odot}$ & $2.3 \pm 0.2$ & $2.3488 \pm 0.0003$ \\
$\log g$ & $3.99 \pm 0.06$ & $3.9860 \pm 0.0001$ \\
\hline
\end{tabular}

asteroseismological observables, we also derive $R / R_{\odot}=2.3 \pm 0.2$ and $\log g=3.99 \pm 0.06$, both values being in agreement with previous determinations (Ryabchikova et al. 2000).

There are two notable issues in this comparison. First, one can see that there is a significant discrepancy between the luminosity derived from asteroseismology and Hipparcos. Matthews et al. (1999) were the first to perform a comparison of the parameters derived by both methods for a sample of roAp stars, including $10 \mathrm{Aql}$ (using the large spacing suggested by Heller \& Kramer 1990). In general, they found a good agreement between the two methods; however, they noted that systematically most Hipparcos luminosities were lower than those ones derived from $\Delta v$, except for $10 \mathrm{Aql}$. Arguing the other way around, they also compared effective temperatures derived using $\Delta v$ and the parallax and compared those with temperatures derived from $\mathrm{H} \beta$ photometry, concluding again a good agreement but "In only one case (10 Aql) does Hipparcos predict a higher temperature." If we assume that indeed the measured parallax is inaccurate and $10 \mathrm{Aql}$ should follow the general trend, then a lower effective temperature and hence a lower luminosity (in agreement with our results) would be needed. Although this explanation is certainly tempting and the deviation noted by Matthews et al. (1999) for $10 \mathrm{Aql}$ is intriguing, the ambiguous nature of our derived fits makes us cautious about coming to any definite conclusions.

Second, the comparison shows that the effective temperature derivation based on spectroscopy tends to cooler values, while photometric calibrations predict a higher temperature (it has to be noted though that the investigation by Kochukhov \& Bagnulo 2006, was aimed solely to study Ap stars in a consistent manner rather than deriving an exact value for $\left.T_{\text {eff }}\right)$. While the asteroseismic value lies in between, it can be seen that only a smaller portion of our models reach into the low temperatures as predicted by spectroscopy (see Fig. 7), and that our best fitting solution does tend to cooler models, considering the grid investigated in this work. If the observed large frequency separation is indeed correct, such a low value for $T_{\text {eff }}$ would present another indication for an overestimated luminosity based on the Hipparcos parallax.

\section{Conclusions}

MOST photometry confirms the rapid pulsation of $10 \mathrm{Aql}$, providing for the first time an unquestionable identification of three pulsation modes in this star. Furthermore, we identify two candidate frequencies with too low a $S / N$ for a clear detection. We do not identify any signal at a frequency previously published in two independent ground-based datasets. We, nonetheless, find that this mode fits well with our present solution and conclude

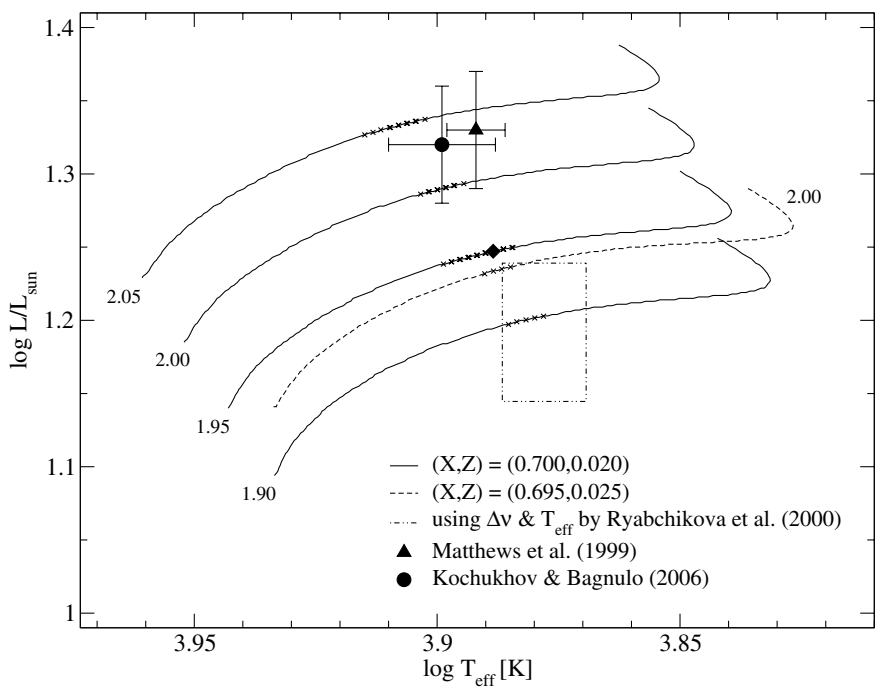

Fig. 7. Theoretical HRD showing previously determined positions of 10 Aql using calibrations based on photometry and the Hipparcos parallax (full circle and triangle) as well as the results derived in this work. Evolutionary tracks correspond to the models presented in Sect. 4.2 (the numbers next to them indicating the corresponding mass). Note that models excluding $\mathrm{He}$ depletion and including envelope convection show no significant deviation from these tracks. The box shows the position derived assuming $\Delta v=50.95 \mu \mathrm{Hz}$, using effective temperatures derived from spectroscopy and an assumption of the mass to be $1.95 \pm 0.05 M_{\odot}$. Small crosses show the position of the models listed in Table 3, with the filled diamond indicating the best model fit using a combination including $f_{i}$.

that it might be evidence for finite mode lifetimes in roAp stars, if the detections are real. Our results confirm previous findings that $10 \mathrm{Aql}$ pulsates with very low amplitudes, and that if additional modes are present their amplitudes must lie significantly below a level of about $40 \mathrm{ppm}$.

Based on the detected signal we can conclude that previously detected amplitude modulation in spectroscopy by Hatzes \& Mkrtichian (2005) was most likely due to beating of unresolved intrinsic frequencies. This is confirmed by simultaneous spectroscopic monitoring, which shows that the observed amplitude modulation in $10 \mathrm{Aql}$ is a pure beating effect (Sachkov et al. 2008).

The MOST light curve presents the longest continuous dataset ever obtained for this star. A time-resolved analysis of the amplitudes for the detected frequencies yields no significant modulation. We conclude that in case the star is not seen poleon, $10 \mathrm{Aql}$ must rotate on timescales longer than a month, confirming previous findings (e.g., Ryabchikova et al. 2005). This is supported by the measured $v \sin i=2.0 \pm 0.5$ (Kochukhov et al. 2002), which, together with the radius derived in this work, yields an approximate lower limit for the inclination of $i>30 \pm 10^{\circ}$.

Our attempt to fit the observed frequencies to theoretical models does not yield an unambiguous solution. We note, however, that models including envelope convection or higher metallicity give a poorer fit. For all frequency combinations fitting within the errors, a $1.95 M_{\odot}$ model without helium depletion yields the most reasonable solution. The derived polar magnetic field strength is about twice the value previously published for the magnetic field modulus, a result that reappears for the MOST observations of $\gamma$ Equ (Gruberbauer et al. 2008). The derived position of $10 \mathrm{Aql}$ deviates by less than $2 \sigma$ from the luminosity based on the Hipparcos parallax. We conclude that this might 
be due to an inaccurate parallax measurement, but caution that our results must be considered with care as discussed in Sect. 4. We also note that the spectroscopic data obtained simulaneously with MOST shows evidence for a pulsation node at a smaller optical depth (i.e., higher in the atmosphere) than considered in the current models (Sachkov et al. 2008). Therefore, the modeling effort and derived results presented in this work must be considered as only a first attempt to understand the pulsation of this star. Still, our results presented here also reflect state of the art modelling efforts for pulsating magnetic stars and indicate the strong need for improved theoretical concepts.

Despite the most complete and precise photometric campaign ever obtained for this star, the oscillation spectrum of 10 Aql remains (compared to other, better studied members such as $\gamma$ Equ or HR 1217) poorly understood. Nevertheless, the MOST observations have certainly shed some new light on this star and provided new insights that might help to unravel the mysteries of this roAp star in future observations and modeling efforts.

Acknowledgements. We are thankful to Mikhail Sachkov, Tanya Ryabchikova, Oleg Kochukhov and Nicole Nesvacil for discussion and collaboration with simultaneous spectroscopy. It is also a pleasure to thank Chris Cameron for valuable discussion on the theory of frequency spacings in roAp stars. D.H., M.G., M.H., W.W.W., T.K., P.R. and R.K. are supported by the Austrian Science Fund (FWF P17580). The Austrian participation in the MOST project is funded by the Austrian Research Promotion Agency (FFG). H.S. is supported by the 21st Century COE programme of MEXT, Japan. D.B.G., J.M.M., A.F.J.M., and S.R. acknowledge funding from the Natural Sciences \& Engineering Research Council (NSERC) Canada. This research has made use of the SIMBAD database, operated at CDS, Strasbourg, France.

\section{References}

Babcock, H. W. 1958, ApJS, 3, 141

Baglin, A., \& The COROT Team. 1998, in New Eyes to See Inside the Sun and Stars, ed. F.-L. Deubner, J. Christensen-Dalsgaard, \& D. Kurtz, IAU Symp., 185,301
Balmforth, N. J., Cunha, M. S., Dolez, N., Gough, D. O., \& Vauclair, S. 2001, MNRAS, 323, 362

Belmonte, J. A., Martinez Roger, C., \& Roca Cortes, T. 1991, A\&A, 248, 541 Belmonte, J. A., Kreidl, T. J., \& Martinez Roger, C. 1992, Inf. Bull. Var. Stars, 3752,1

Bigot, L., \& Dziembowski, W. A. 2002, A\&A, 391, 235

Bigot, L., \& Weiss, W. W. 2002, Commun. Asteroseismol., 141, 26 Cunha, M. S. 2006, MNRAS, 365, 153

Gruberbauer, M., Saio, H., Huber, D., et al. 2008, A\&A, 480, 223

Guenther, D. B., \& Brown, K. I. T. 2004, ApJ, 600, 419

Guenther, D. B., Demarque, P., Kim, Y.-C., \& Pinsonneault, M. H. 1992, ApJ, 387, 372

Hatzes, A. P., \& Mkrtichian, D. E. 2005, A\&A, 430, 279

Heller, C. H., \& Kramer, K. S. 1990, MNRAS, 244, 372

Huber, D., \& Reegen, P. 2008, Commun. Asteroseismol., 152, 77

Iglesias, C. A., \& Rogers, F. J. 1996, ApJ, 464, 943

Kallinger, T., Reegen, P., \& Weiss, W. W. 2008, A\&A, 481, 571

Kochukhov, O. 2004, ApJ, 615, L149

Kochukhov, O. 2006, A\&A, 446, 1051

Kochukhov, O., \& Bagnulo, S. 2006, A\&A, 450, 763

Kochukhov, O., Landstreet, J. D., Ryabchikova, T., Weiss, W. W., \& Kupka, F. 2002, MNRAS, 337, L1

Kurtz, D. W. 1978, Inf. Bull. Var. Stars, 1436, 1

Kurtz, D. W. 1982, MNRAS, 200, 807

Lenz, P., \& Breger, M. 2004, in The A-Star Puzzle, ed. J. Zverko, J. Ziznovsky, S. J. Adelman, \& W. W. Weiss, IAU Symp., 224, 786

Matthews, J. M., Kurtz, D. W., \& Martinez, P. 1999, ApJ, 511, 422

Preston, G. W. 1970, PASP, 82, 878

Reegen, P. 2007, A\&A, 467, 1353

Reegen, P., Kallinger, T., Frast, D., et al. 2006, MNRAS, 367, 1417

Rowe, J. F., Matthews, J. M., Kuschnig, R., et al. 2006, Mem. Soc. Astron. Ital., 77,282

Ryabchikova, T. A., Savanov, I. S., Hatzes, A. P., Weiss, W. W., \& Handler, G. 2000, A\&A, 357, 981

Ryabchikova, T., Wade, G. A., Aurière, M., et al. 2005, A\&A, 429, L55

Sachkov, M., Kochukhov, O., Ryabchikova, T., et al. 2008, Contributions of the Astronomical Observatory Skalnate Pleso, accepted [arXiv: 0712.1340]

Saio, H. 2005, MNRAS, 360, 1022

Saio, H., \& Gautschy, A. 2004, MNRAS, 350, 485

Tassoul, M. 1980, ApJS, 43, 469

Walker, G., Matthews, J., Kuschnig, R., et al. 2003, PASP, 115, 1023 